\title{
An improved heteroduplex analysis for rapid genotyping of SNPs and single base pair indels
}

\author{
Jiangbo Fan ${ }^{1,2}$, Ye Xia*, \& Guo-Liang Wang ${ }^{\star \star, 1,2}$
}

\section{ABSTRACT}

SNPs and single base pair (SBP) insertion/ deletions (indels) are not only the most abundant genetic markers for genetic mapping and breeding selection, but also always occur in the mutants generated from chemical mutagenesis or CRISPR/ Cas9-mediated genome editing. Most of the current SNP and SBP indel genotyping methods are time-consuming and/or require special equipment or reagents. Here, we describe an improved heteroduplex analysis method, named iHDA, that can readily discriminate SNP and SBP indel alleles with specially designed DNA probes that harbor a couple of nucleotides adjacent to the SNP site. By hybridizing with the same probe, SNP and SBP indel alleles form different heteroduplexes, differing in bulge size, which show different mobility on a polyacrylamide gel. Therefore, iHDA is an easy, fast and inexpensive method for SNP and SBP indel genotyping.

\section{METHOD SUMMARY}

An improved heteroduplex analysis method, named iHDA, takes advantage of a specially designed probe, which possesses three to six inserted nucleotides adjacent to a SNP or single base pair (SBP) indel site. The method discriminates mutant alleles making SNP and SBP indel genotyping easier, cheaper and faster.

\section{KEYWORDS \\ CRISPR $\cdot$ genotyping $\cdot$ heteroduplex assay - iHDA - improved hetero- duplex assay - single base pair insertion/deletion $\cdot$ single nucleotide polymorphism}

'Department of Plant Pathology, The Ohio State University, Columbus, $\mathrm{OH} 43210$, USA; ${ }^{2}$ State Key Laboratory for Biology of Plant Diseases \& Insect Pests, Institute of Plant Protection, Chinese Academy of Agricultural Sciences, Beijing 100193, China; *Author for correspondence: xia.374@osu.edu; **Author for correspondence: wang.620@osu.edu

BioTechniques 67: 6-10 (July 2019) 10.2144/ btn-2019-0012

Vol. 67 | No. 1 | (c) 2019 Guo-Liang Wang

\section{GRAPHICAL ABSTRACT}
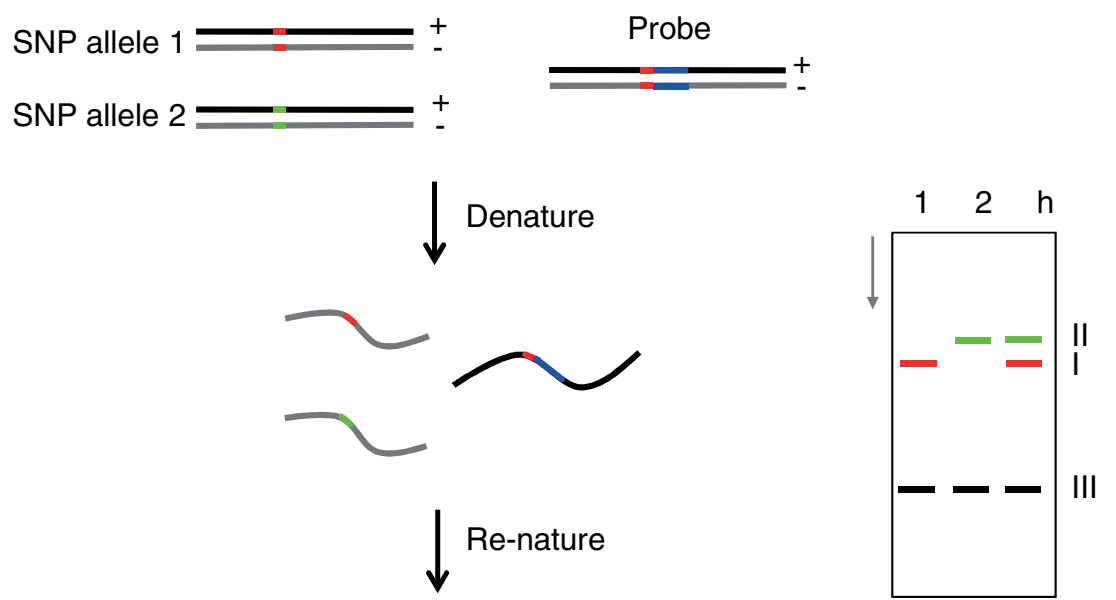
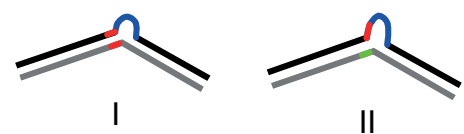

SNPs are the most abundant and densest genetic variations in plant and animal genomes [1-5]. SNPs have recently become the most important genetic markers for genome-wide association studies, finemapping and cloning of genes of interest, and breeding selection. Small indels are another type of genetic marker abundant in genomes with single base pair (SBP) insertions/deletions (indels) and monomeric base pair extensions are the most common class [6,7]. In addition to occurring in natural populations, SNPs and SBP indels always occur in mutants generated by chemical mutagens such as ethyl methanesulfonate (EMS) or by CRISPR/Cas9-based genome editing. EMS mutagenisis converts $\mathrm{C} / \mathrm{G}$ to $T / A$ base pairings and sometimes generates SBP indels, while CRISPR/Cas9-mediated genome editing often produces a point mutation or an SBP indel in the target site [8-10]. Recent research shows that cytidine deaminases or adenine deaminases can be fused to Cas9 or Cpf1 (newly discovered class 2/type V CRISPR-Cas DNA endonuclease) to function as base editors that can convert G-C to A-T or vice versa in plants [11-17]. Identification of these mutations - single nucleotide substitution or SBP indels - in transgenic plants is timeconsuming and expensive. Therefore, development of a rapid and accurate method for detecting SNPs or SBP indels is important for many basic and applied projects.

SNP genotyping methods can be classified into three major types: DNA hybridization-based, enzyme-based, and confirmation- or melting temperature-based methods [18-20]. Hybridization-based methods include dynamic allele-specific hybridization, molecular beacons, SNP arrays and a probes-oncarrier DNA chip [18-21]. Enzyme-based methods include restriction fragment length polymorphism (RFLP) and cleaved amplified polymorphic sequence (CAPS) assay, invader assay, primer extension assay, TaqMan assay, oligonucleotide ligation assay (OLA), Surveyor nuclease assay, T7 endonuclease I (T7EI) cleavage assay, PCR/RNP assay, and specific highsensitivity enzymatic reporter unlocking (SHERLOCK) assay $[22,23]$. The conformation- or melting temperature-based 


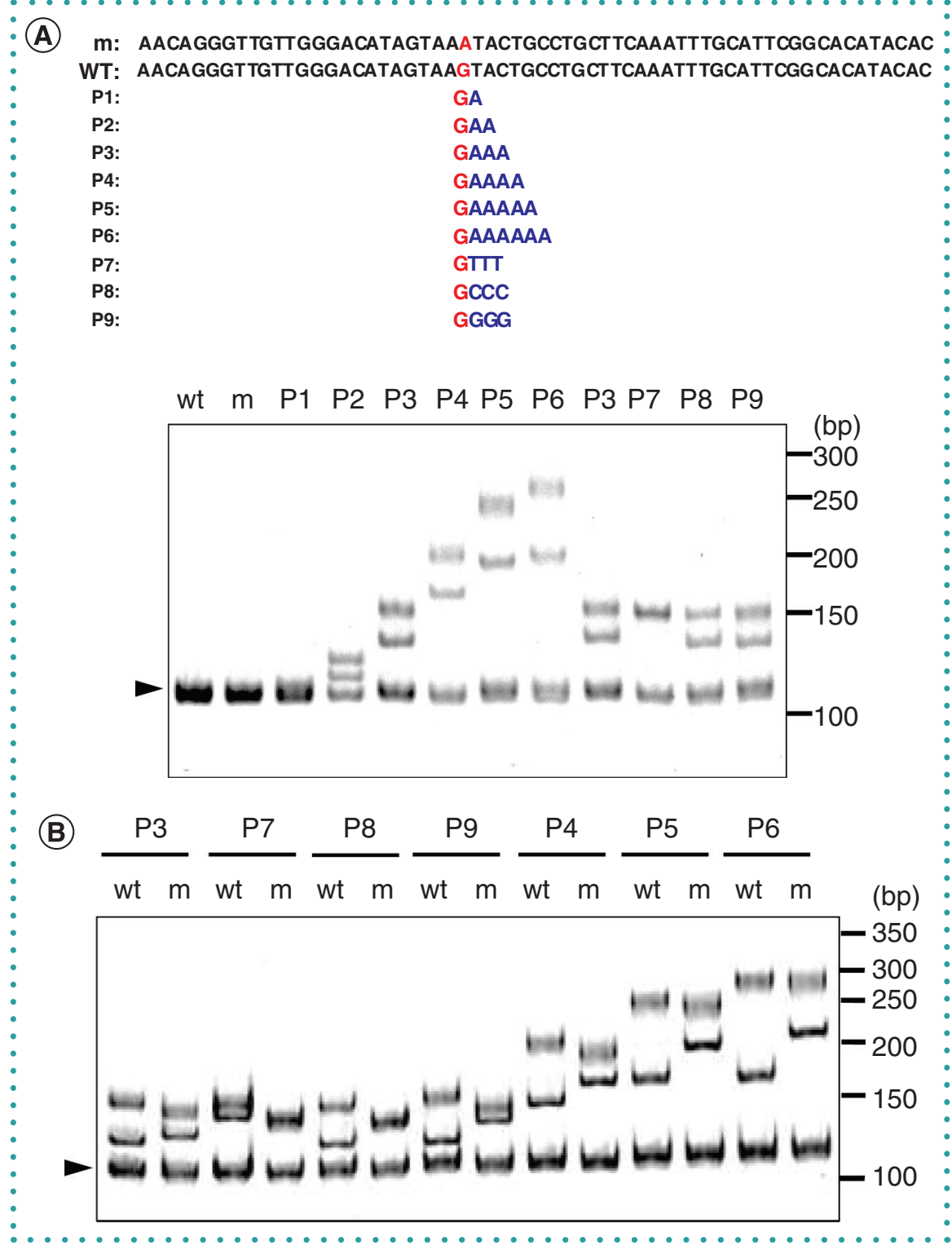

Figure 1. Effects of bulge size and composition on heteroduplex mobility and application of iHDA for the discrimination of SNP alleles. (A) Sequences of the WT (IR64) and the $m$ allele (sds2) and the nine probes (upper panel). The nucleotides in blue were introduced after the SNP nucleotide $\mathrm{G}$ in the probes. The lower panel shows the heteroduplex formation obtained with the nine probes in the upper panel. WT amplicons were mixed with the probes and were subjected to a denaturation-renaturation cycle. Probes are labeled on the top. WT and $\mathrm{m}$ fragments were also used as the probe in lane wt and $\mathrm{m}$, respectively. The samples were separated on a continuous $8 \%$ PAGE gel. DNA markers are labeled on the right. (B) The heteroduplex bands formed in the WT (IR64) and $\mathrm{m}$ (sds2) samples with seven probes. The samples were separated on a continuous $8 \%$ PAGE gel. Probe information is provided in part A (lower panel). DNA markers are labeled on the right.

m: Mutant; WT: Wild-type.

$\checkmark$ methods include single-strand conformation polymorphism assay, heteroduplex assay (HDA) or heteroduplex mobility assay (HMA), denaturing and temperature gradient gel electrophoresis (DGGE and TGGE) assay, denaturing high-performance liquid chromatography (DHPLC), high-resolution melting analysis (HRMA), and MutS binding

Vol. 67 | No. 1 | ๑) 2019 Guo-Liang Wang
For example, PCR/RNP assay, SHERLOCK assay, and a probes-on-carrier DNA chip have recently been used for SNP genotyping [21-23]. In this report, we took advantage of HDA to develop a new method to facilitate SNP genotyping. Heteroduplexes are formed from the hybridization of heterologous sequences, usually allelic sequences with SNPs or indels. Unmatched sequences or sites in the homologous region form bulges that sharply reduce heteroduplex mobility. The heteroduplex-based analysis for DNA mutation detection is thus named HDA or HMA, which enhances the differences between indel alleles, and has been applied to detect genome variations including indels and point mutations [28-30]. When used to discriminate SNP alleles, HDA requires a special solvent system in order to increase the heteroduplex mobility shift [31]. To aid the genotyping of SNP alleles, here we describe an improved HDA method, named HDA, that can readily discriminate SNP alleles with a specially designed DNA probe, making it an easy, fast and inexpensive method for SNPs genotyping. In addition, iHDA is effective for detecting SBP indels. Because this method does not require any special equipment, enzymes or proteins, and has no sequence limitations, it can be easily adopted for routine SNP and SBP indel genotyping in most laboratories.

\section{MATERIALS \& METHODS \\ PCR amplification}

PCR reactions were carried out with a commercial premix (Tsingke Inc., Beijing, Cat \#: TSE004) following the manufacturer's instructions. If an Ex Taq DNA polymerase (Takara, Cat \#: RR006A) was used, the $20-\mu$ l reaction mix contained $1 \mathrm{X}$ buffer, $0.5 \mu \mathrm{M}$ of each primer, $0.2 \mathrm{mM}$ dNTP of each nucleotide, 20-100 ng of rice template DNA, and/or $0.01-0.05 \mathrm{pg}$ of probe DNA and 0.5U Taq polymerase. The probe was produced by PCR (see the section on probe design and preparation for more information). PCR amplification was conducted as follows: $95^{\circ} \mathrm{C} 2 \mathrm{~min}$, then an amplification cycle (denature at $94^{\circ} \mathrm{C}$ for $20 \mathrm{~s}$, anneal at $50-60^{\circ} \mathrm{C}$ for $20 \mathrm{~s}$, extension at $72^{\circ} \mathrm{C}$ for $15 \mathrm{~s}$ ) for 32 cycles, $72^{\circ} \mathrm{C}$ for $6 \mathrm{~min}$, then $16^{\circ} \mathrm{C}$ or terminate the reaction. The PCR amplification was carried out with a T100 Thermal Cycler (Bio-Rad). Primers for amplification of the target gene region were designed to amplify approximately a 120-bp 
fragment. The primers used in this study are listed in Supplementary Table S1. All the primers (including FAM-labeled) were synthesized by Tsingke, Inc.

\section{Probe design \& preparation}

The probe used in iHDA is a sequence that contains artifically inserted nucleotides adjacent to the SNP or SBP indel site (Figure 1A). The probe was produced by overlapping PCR with four primers: primer 1 , 2, 3 and 4. Primers 1 and 2 are the forward and reverse primer of the target gene, respectively. Primers 3 and 4 are reverse complementary primers that span the SNP or SBP indel site and harbor inserted nucleotides adjacent to the SNP or SBP indel site. The probe was generated by two runs of PCR amplifications. First PCR amplification produces two overlapping fragments, $\mathrm{F} 1$ and $F 2$. Then, $F 1$ and $F 2$ were purified from agarose gel by gel slicing. Purified F1 and F2 were mixed and used as the template for the second PCR with primers 1 and 2. The products of the second PCR amplification were the iHDA probe with inserted nucleotides. The FAM-labeled probe used for the quantification of SNP alleles with the bulked sample was generated in the same way with a FAM-labeled primer, primer 1 . The probes were confirmed by sequencing before use in the study.

\section{Heteroduplex generation}

Heteroduplexes were formed using two methods. In the first method, the amplification of the target gene and the probe were performed in separate tubes and then equal volumes of the reaction solutions were mixed and underwent a denature-renature cycle, which was performed in a T100 Thermal Cycler (Bio-Rad) with the following conditions: denaturation at $95^{\circ} \mathrm{C}$ for $5 \mathrm{~min}$ and then renaturation at $70^{\circ} \mathrm{C}$ for $10 \mathrm{~min}$. In the second method, the prepared probe $(0.01-0.05 \mathrm{pg})$ was added to the PCR reaction mixture of the target gene (with 20-100 ng rice genomic DNA as template). The mixture underwent a standard PCR amplification as mentioned above. Since the PCR program included a denature-renature cycle at the end of the PCR cycles, heteroduplexes between the target gene amplicon and the probe were formed.

\section{Cast \& gel running of PAGE}

The concentration of continuous PAGE gels was $8 \%$. PAGE gels were prepared with a Bio-Rad western blot plate $(12.5 \mathrm{~cm} \times 6.5 \mathrm{~cm} \times 1.0 \mathrm{~mm})$. The gel contained $23.6 \mathrm{ml}$ of water, $8 \mathrm{ml}$ of $5 X$ TBE buffer, $8 \mathrm{ml}$ of $40 \%$ acrylamide (acrylamide:bis-acrylamide $=29: 1), 400 \mu \mathrm{l}$ of $10 \%$ ammonium persulfate, and $40 \mu \mathrm{l}$ of TEMED. The total volume of $40 \mathrm{ml}$ was sufficient for six plates $(12.5 \mathrm{~cm} \times 6.5 \mathrm{~cm} \times 1.0 \mathrm{~mm})$. After the gels are prepared, the samples can be loaded for standard electrophoresis. Before they were loaded into wells, loading buffer containing SYBR Green I dye (Invitrogen, Cat \#: S7563) was added to the samples. The SYBR Green I dye was added to the sample at a final concentration of $1 X$, which originally was 10000X. For each sample, $2 \mu$ l of generated heterduplexes (around 50-100 ng) were loaded to the PAGE gel. The PAGE gels were run at $120-150$ volts for $45-60$ min with $1 X$ TBE electrophoresis buffer. After electrophoresis, the gel can be directly visualized with the Bio-Rad ChemiDoc XRS+ system, because SYBR Green I dye has already been added and associated with DNA molecules. For the quantification of SNP alleles in the bulked sample with FAM-labeled probes, SYBR Green I dye was not added.

\section{RESULTS \& DISCUSSION}

Effects of bulge size \& composition on heteroduplex mobility

It was reported that both bulge size and composition affect heteroduplex mobility [20]. To figure out how bulge size and composition affect heteroduplex mobility, we carried out an iHDA assay with a series of artificial probes. By inserting a few nucleotides adjacent to the SNP site, which keeps the same as one allele (here is the wild-type [WT] allele), we constructed a series of probes (P1-6) that harbor one to six additional nucleotides of adenine $(A)$, that is, $1 A$ to $6 A$ s, and different nucleotides with the same number, that is, 3A, 3T, 3C and 3G (Figure 1A, top panel). The target PCR amplicon (approximately $150 \mathrm{bp}$ ) spanning the SNP site was mixed with the probe (same size as the PCR amplicon except the inserted sequences), and the mixture was subjected to a denature-renature cycle in a PCR machine before loading on a PAGE gel. When the number of added A nucleotides was increased, the heteroduplex mobility sharply decreased (Figure 1A, bottom panel). The formation of the two types of heteroduplexes (two bands) was due to the sequence difference in the bulges. Similarly, probes $\mathrm{P} 3, \mathrm{P} 7, \mathrm{P} 8$ and $\mathrm{P} 9$, which had the same bulge size (three nucleotides), but with different bulge compositions (A, T, C or $G$ ), also produced different band patterns, supporting the notion that bulge composition influences heteroduplex DNA mobility. It is noteworthy that probe $\mathrm{P} 1$ with one additional A nucleotide did not show any mobility shift band compared with the WT probe but that probe P2 did produce two heteroduplex bands, suggesting that at least two additional nucleotides are needed in the probe to produce obvious mobility shift.

Based on the above findings, SNP alleles could be discriminated with a common probe that harbors several ( $n$ ) nucleotides inserted adjacent to the SNP site of one allele (for example the WT allele). To test whether the above probes can be used to distinguish different alleles, we hybridized seven probes (P3-9) in Figure 1A with the WT (IR64) and mutant ( $\mathrm{m}$; sds2) fragments that differed by only one SNP (sequence in the top panel of Figure 1A). The results showed that WT and $m$ alleles produced different patterns of bands for all of the probes used (Figure 1B).

\section{SNP genotyping with iHDA}

To validate the effectiveness of iHDA for the genotyping of SNP alleles, we genotyped several selected individuals with known genotypes (alleles in Figure 1A, WT and m) with probe $\mathrm{P} 6$. The results showed that the genotypes of the WT, $m$, and their hybrid $(\mathrm{H})$ were clearly determined and were consistent with their genotypes (Figure 2A). In this assay, WT and $m$ sequences produce two heteroduplex bands, respectively, with two of them not able to be discriminated. Thus, the hybrid samples produced only three bands. All the band patterns were consistent with their genotypes.

We also used iHDA to detect a 1-bp deletion (SBP indel). As shown in Figure 2B, the genotypes of the 14 samples of one indica (IR64, I) allele, one japonica (TP309, J) allele, and their mixture ( $50 \%$ each to mimic $\mathrm{H}$ ) were clearly determined by a probe with three Adenines inserted adjacent to the deletion site. The results demonstrated that the indica (I) allele and japonica $(\mathrm{J})$ allele produced two heteroduplex bands, respectively, with all of them distinguishable from each other. The hybrid samples produced four heteroduplex bands corresponding to that of the I and 


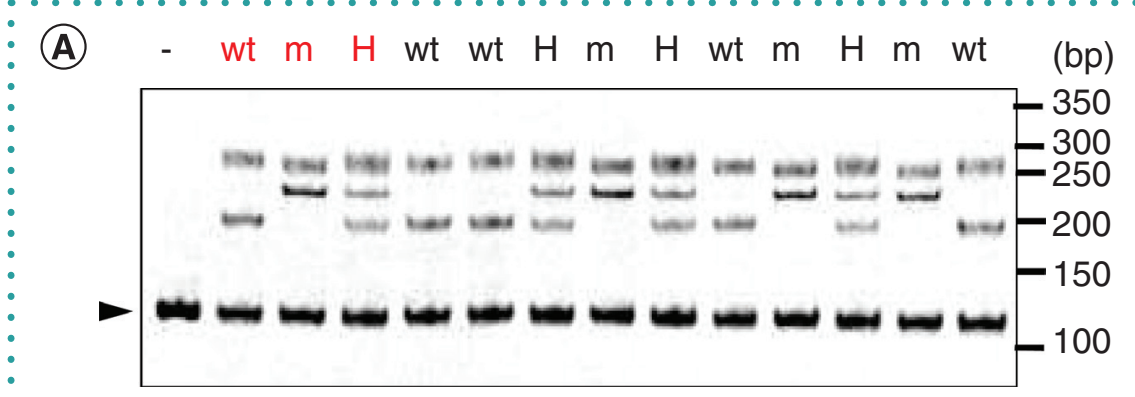

(B)

Japonica: GCAATAGGAGACTATTCTTTTGGGATG--GATCCAAATGAGAAGGGTGATTT Indica: GCAATAGGAGACTATTCTTTTGGGATGAGATCCAAATGAGAAGGGTGATTT $\checkmark$

Probe: GCAATAGgAgACTATTCTTTTGgGATGAAAAGATCCAAATGAGAAGgGTGATTT

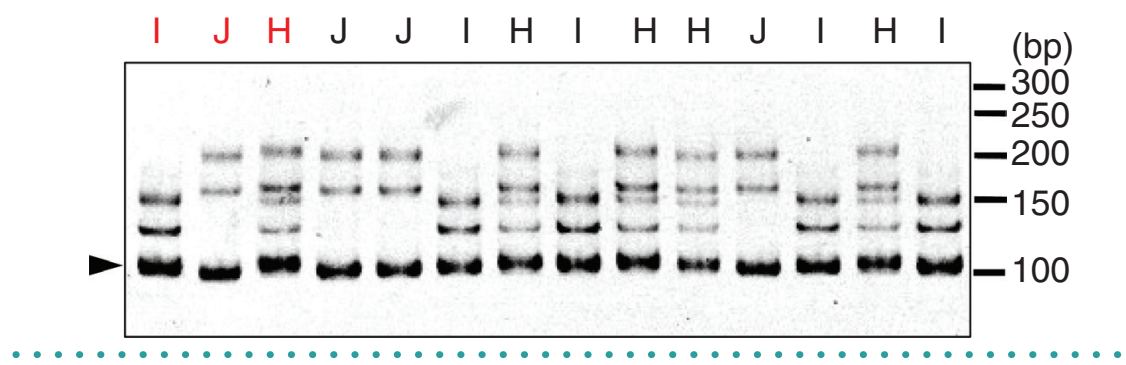

Figure 2. Two cases of SNP and single base pair indel genotyping with iHDA. (A) A case study of genotyping to distinguish homozygous and heterozygous samples. '-' indicates that no genomic DNA was added. WT, m, and H indicate WT (IR64), mutant (sds2), and a mixture of equal amounts of IR64 and sds2 DNA (mimic hybrid). The 2nd to 4th lanes indicate control samples with known genotypes as labeled in red. The samples were separated on an $8 \%$ PAGE gel. DNA markers are labeled on the right. (B) A case study of genotyping an single base pair indel (red A). The sequences of indica (IR64) and japonica (TP309) alleles and the iHDA probs are shown on the top of gel picture. The three introduced A nucleotides are labeled in blue. Lanes $\mathrm{I}, \mathrm{J}$ and $\mathrm{H}$ indicate indica, japonica, and an equal mix of both genotypes (mimic hybrid), respectively. The first three lanes $(\mathrm{I}, \mathrm{J}$ and $\mathrm{H}$ ) represent control samples with known genotypes as labeled in red. The samples were separated on an $8 \%$ PAGE gel. DNA markers are labeled on the right.

H: Hybrid; I: Indica; J: Japonica; m: Mutant; WT: Wild-type.

J alleles. The results demonstrate that iHDA readily discriminates SBP indels. Since the bulge size and composition affect the mobility of heteroduplexes, each SBP indel can form two heteroduplexes that differ in their bulge nucleotide compositions. In the homozygote lane (I and $\mathrm{J}$ ), there were three bands, including two heteroduplex bands and one homoduplex band (the probe and the target were indiscriminable here). In the heterozygote lane $(H)$, there were five bands, including four heteroduplex bands and one homoduplex band (the probe and the two targets were indiscriminable). It is noteworthy that sometimes heteroduplexes with the same bulge size but different compositions were not separable in the PAGE gel, such as $\mathrm{P} 7$ and $\mathrm{P} 8$ with the WT allele in Figure 1B.
iHDA can be applied for quantification of SNP alleles

To demonstrate whether iHDA can be used for the quantification of SNP alleles, we made several artificial samples by mixing WT and sds2 DNA in different ratios. SDS2 amplicons generated from the samples were mixed with fluorescein (FAM)-labeled probe (prepared by PCR amplification with a FAM-labeled primer), subjected to a denature-renature cycle, and then separated on a PAGE gel. The results demonstrated that the two SNP alleles (WT and sds2) were clearly separated and that the intensity of $s d s 2$ - and WT-specific bands on the gel was well correlated with the corresponding quantity of input DNA (Figure 3). FAM-labeled primer rather than SYBR Green I dye was used to visualize allelespecific heteroduplex bands because the intensity of bands was proportional to the relative amount of each allele in the mixture.

Compared with traditional HDA, iHDA shows the ability to discriminate SNP alleles and SBP indel alleles, which is beyond the ability of traditional HDA. In this report, our results show that iHDA, which involves the addition of a few nucleotides adjacent to SNP sites in the probes, is highly effective for discriminating SNP and SBP indel alleles. iHDA has at least five advantages compared with other available methods. First, unlike the invader assay, primer extension assay, TaqMan assay, OLA, TGGE/DGGE, DHPLC and HRMA, iHDA does not require any special equipment. Second, unlike RFLP and CAPS, MutS binding assay, OLA, T7EI cleavage assay, Surveyor nuclease assay, PCR/RNP assay and SHERLOCK assay, iHDA does not require special enzymes or proteins. Third, unlike RFLP, PCR/RNP assay and SHERLOCK assay, which require specific enzyme sites or PAM sites, iHDA has no sequence limitation. iHDA depends on conformational changes rather than on specific sequence differences, making it a general method to detect any SNP alleles or SBP indels. Fourth, iHDA is easy, fast and inexpensive, requiring only PCR amplification and PAGE, which are available in most laboratories. Finally, iHDA can be used to quantify SNP alleles in bulked samples and to quantify any other nucleotide substitutions, such as A-to-I RNA editing in heterogeneous samples.

\section{FUTURE PERSPECTIVE}

Detection of SNP and SBP indel variations has become a routine technique in many laboratories. The iHDA method can be carried out in one tube for genotyping of SNPs and SBP indels in a target region. iHDA can be automated with a capillary electrophoresis system and a PCR machine to achieve a highthroughput screening of large genetic materials.

\section{AUTHOR CONTRIBUTIONS}

$J F, Y X$ and GLW designed the experiments. JF performed the experiments. JF and GLW wrote the paper.

\section{ACKNOWLEDGMENTS}

The authors thank Dr Zhiqiang Li (Institute of Plant Protection, Chinese Academy of Agricultural Sciences) for the preparation of rice seedlings used in this study. 


\section{FINANCIAL \& COMPETING \\ INTERESTS DISCLOSURE}

This study was supported by grants from the Ohio Agricultural Research and Development Center (OARDC) and the Institute of Plant Protection, Chinese Academy of Agricultural Sciences. The authors have no other relevant affiliations or financial involvement with any organization or entity with a financial interest in or financial conflict with the subject matter or materials discussed in the manuscript apart from those disclosed.

No writing assistance was utilized in the production of this manuscript.

\section{OPEN ACCESS}

This work is licensed under the AttributionNonCommercial-NoDerivatives 4.0 Unported License. To view a copy of this license, visit http://creativecommons.org/licenses/by-ncnd/4.0/

\section{SUPPLEMENTARY DATA}

To view the supplementary data that accompany this paper please visit the journal website at: www.future-science.com/doi/ suppl/10.2144/btn-2019-0012

\section{REFERENCES}

1. Alonso-Blanco C, Andrade J, Becker C et al. 1,135 genomes reveal the global pattern of polymorphism in Arabidopsis thaliana. Cell 166(2), 481-491 (2016)

2. The International HapMap Consortium. A second generation human haplotype map of over 3.1 million 1 (2007)

3. Wang W, Mauleon R, Hu Z et al. Genomic variation in 3,010 diverse accessions of Asian cultivated rice. Nature 557(7703), 43-49 (2018).

4. Srinivasan $\mathrm{S}$, Clements JA, Batra J. Single nucleotide Crit. Rev. Clin. Lab Sci. 53(1), 29-39 (2016).

5. Marcotuli I, Gadaleta A, Mangini G et al. Development of a high-density SNP-based linkage map and detection of QTL for beta-glucans, protein content, grain yield per spike and heading tine
Sci. 18(6), 1329 (2017).

6. Jain A, Roorkiwal M, Kale S, Garg V, Yadala R, Varshney RK. InDel markers: an extended marker resource for molecular breeding in chickpea. PLoS One 14(3) e0213999 (2019).

7. Mills RE, Luttig CT, Larkins CE et al. An initial map of genome. Genome Res. 16(9), 1182-1190 (2006).

8. Nekrasov V, Staskawicz B, Weigel D, Jones JD, Kamoun S. Targeted mutagenesis in the model plant Nicotiana benthamiana using Cas9 RNA-guided endo
Nature Biotechnol. 31(8), 691-693 (2013).

9. Shan $Q$, Wang $Y$, Li J et al. Targeted genome modification of crop plants using a CRISPR-Cas system. Natu Biotechnol. 31(8), 686-688 (2013).

10. Kim Y, Schumaker KS, Zhu JK. EMS mutagenesis of Arabidopsis. Methods Mol. Biol. 323, 101-103 (2006).

11. Kim JS. Precision genome engineering through adenine and cytosine base editing. Nature Plants 4(3), 148-151

12. Li C, Zong $Y$, Wang $Y$ et al. Expanded base editing in rice and wheat using a Cas9-adenosine deaminase fusion. Genome Biol. 19(1), 59 (2018).

13. Li X, Wang Y, Liu Y et al. Base editing with a Cpf1-cytidine deaminase fusion. Nature Biotechnol. 36(4), 324327 (2018).

14. Lu Y, Zhu JK. Precise editing of a target base in the rice
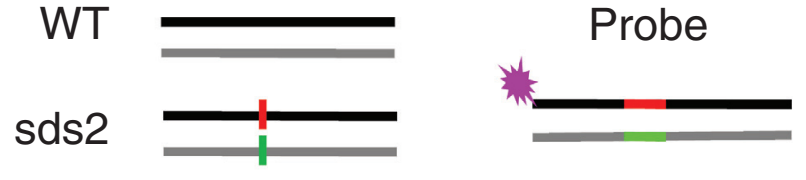

$\begin{array}{cccccccc}\text { sds2 } & 0 & 20 & 40 & 60 & 80 & 100 & (\%) \\ \text { WT } & 100 & 80 & 60 & 40 & 20 & 0\end{array}$

(\%)

(\%)

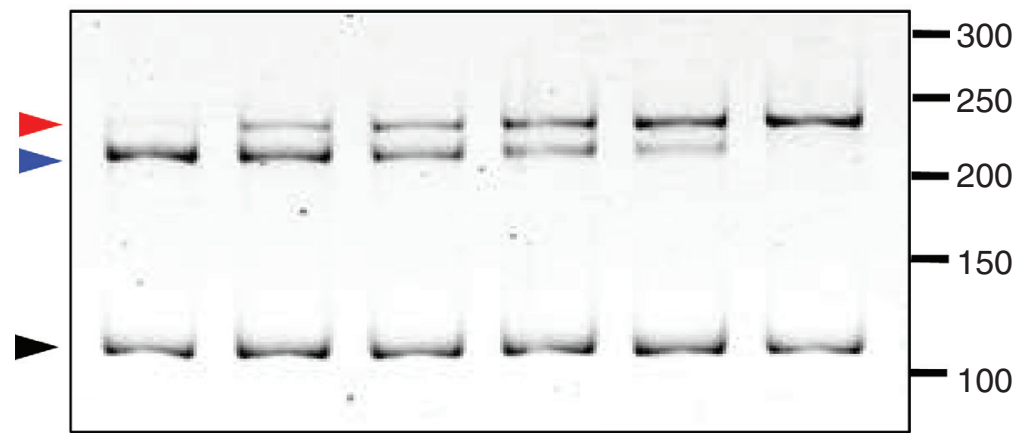

$\begin{array}{rrrrrrr}\text { sds2 } & 0.06 & 0.26 & 0.43 & 0.60 & 0.75 & 0.96 \\ \text { WT } & 0.94 & 0.74 & 0.57 & 0.40 & 0.25 & 0.04\end{array}$

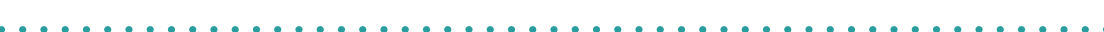

Figure 3. Application of improved heteroduplex analysis method for quantification of SNP alleles. Different ratios of two SNP alleles were used in combination with a fluorescent probe. The SNP alleles (the WT IR64 and the mutant sds2) and the fluorescein-dye labelled probe (purple asterisk) are shown on the top. The proportion of each allele is indicated on the top of the gel photograph. Arrows indicate the WT band (blue), sds2 band (red), and probe band (black). The green and red bars in sds2 allele and in probe indicate SNP site and insertion respectively. The samples were separated on an $8 \%$ PAGE gel. DNA markers are labeled on the right. The quantification of sds2 and WT allelespecific heterduplexes were determined by Bio-Rad ImageLab 6.0 software and the relative values were labeled below the picture, which is well correlated with the ratio of each allele. WT: Wild-type.

genome using a modified CRISPR/Cas9 system. Molecu523-525 (2017).

15. Ren B, Yan F, Kuang $Y$ et al. Improved base editor for efficiently inducing genetic variations in rice with CRISPR/ Cas9-guided hyperactive hAID mutant. Molecular Plant 11(4), 623-626 (2018).

16. Yan F, Kuang Y, Ren B et al. Highly efficient A.T to G.C base editing by Cas9n-guided tRNA adenosine dean

17. Richaud $D$, Stange $C$, Gadaleta $A$ et al. Identification of lycopene epsilon cyclase (LCYE) gene mutants to potentially increase $\beta$-carotene content in durum wheat One 13(12), 0208948 (2018).

18. Komar A (Ed.). Single Nucleotide Polymorphisms: Methods and Protocols. Humana Press (2009).

19. Kwok $P(E d)$. Single nucleotide polymorphisms: methods and protocols. Humana Press (2003).

20. Patrinos GP, Danielson PB, Ansorge WJ. Chapter $1-$ Molecular diagnostics: past, present, and future. In Molecular Diagnostics (Third Edition), Patrinos GP (Ed.).

21. Saifullah, Fuke $\mathrm{S}$, Nagasawa $\mathrm{H}$ et al. Single nucleotide recognition using a probes-on-carrier DNA chip. BioTechniques 66(2), 73-78 (2019).

22. Gootenberg JS, Abudayyeh 00 , Kellner MJ et al. Multiplexed and portable nucleic acid detection platform with Cas13, Cas12a, and Csm6. Cas13, Cas12a, and Csm6.

23. Liang Z, Chen $K$, Yan $Y$ et al. Genotyping genome-edited mutations in plants using CRISPR ribonucleopro2062 (2018).

24. Bovina R, Brunazzi A, Gasparini G et al. Developmen of a TILLING resource in durum wheat for reverse- and forward-genetic analyses. BIOONE 65 (2014).

25. Pilato B, De Summa S, Danza K et al. DHPLC/SURVEYOR nuclease: a sensitive, rapid and affordable method to analyze $B R C A 1$ and $B R C A 2$ mutations in breast cancer families. Mol. Biotechnol. 52(1), 8-15 (2012).

26. Colasuonno P, Incerti O, Lozito ML et al. DHPLC technology for high-throughput detection of mutations in a durum wheat TILLING population. BMC Genetics 17, 43 (2016).

27. Hestekin CN, Barron AE. The potential of electrophoretic mobility shift assays for clinical mutation detection. Electrophoresis 27(19), 3805-3815 (2006)

28. Sgourou A, Papachatzopoulou A, Katsila T et al. Chapter 3 - Low- and medium-throughput variant detection methods: a historical perspective. In: Molecular Diagnostics (Third Edition), Patrinos GP (Ed.). Academic Press, 23-39 (2017).

29. Nakagawa $Y$, Yamamoto $T$, Suzuki $K$ et al. Screening methods to identify TALEN-mediated knockout mice. Exp. Animals 63(1), 79-84 (2014).

30. Naert T, Vleminckx K. Genotyping of CRISPR/Cas9 genome edited Xenopus tropicalis. In: Xenopus: Methods and Protocols. Vleminckx K (Ed.). Springer, NY, USA 67-82 (2018)

31. Ganguly A, Rock MJ, Prockop DJ. Conformation-sensitive gel electrophoresis for rapid detection of single-base differences in double-stranded PCR products and DNA fragments: evidence for solvent-induced bends in DNA heteroduplexes. Proc. Natl Acad. Sci. USA 90(21), 10325 (1993). 